\title{
ASO Author Reflections: "Right-Sizing" Radiation Therapy After Mastectomy
}

\author{
Anees B. Chagpar, MD, MSc, MPH, MA, MBA \\ Department of Surgery, Yale University School of Medicine, New Haven, CT
}

\section{PAST}

Historically, patients with mastectomy were told they would not require radiation therapy - indeed, it was one of the considerations for opting for mastectomy over breast conserving approaches. With the emergence of the Denmark and British Columbia trials demonstrating a survival advantage to post-mastectomy radiation therapy (PMRT) for node positive patients $^{1,2}$ and a plethora of data to suggest that PMRT reduces local recurrence rates and breast cancer specific mortality, ${ }^{3}$ practitioners became much more liberal in their use of this modality. Indeed, major consensus panels advocated for the same. ${ }^{4}$ But radiation is not without toxicity-both physical and financial — and so the question arises, can we tailor the use of radiation therapy such that it is given only to those patients who will truly benefit from it?

\section{PRESENT}

With the increasing use of genomic assays and neoadjuvant therapy to predict prognosis, some wonder about whether decisions regarding PMRT could be personalized to individual patients. In an effort to reduce toxicity, there is currently a move to de-escalate therapy from both a surgical as well as a radiation therapy perspective, particularly when it comes to management of the axilla. Lymphedema remains a concern for many patients with overly aggressive treatment but the need for optimal local control weighs on the minds of many multidisciplinary teams. The debate continues as to

(C) Society of Surgical Oncology 2021

First Received: 19 July 2021

Accepted: 21 July 2021

Published Online: 9 August 2021

A. B. Chagpar, MD, MSc, MPH, MA, MBA

e-mail: anees.chagpar@yale.edu which modality can provide optimal local control while minimizing toxicity. ${ }^{5}$ While both surgeons and radiation oncologists seem keen to do less, avoiding both modalities may compromise oncologic control.

\section{FUTURE}

Clinical trials are ongoing that hope to provide some data to answer the question of how we can "right size" local therapy for individual patients. Until data from these trials can provide insight to guide our management, there remains a delicate balance between the risks and the benefits of PMRT in T1/2N1 breast cancer patients, and the debate over these issues persists.

\section{REFERENCES}

1. Ragaz J, Olivotto IA, Spinelli JJ, et al. Locoregional radiation therapy in patients with high-risk breast cancer receiving adjuvant chemotherapy: 20-year results of the British Columbia randomized trial. J Natl Cancer Inst. 2005;97(2):116-26.

2. Overgaard M, Nielsen HM, Overgaard J. Is the benefit of postmastectomy irradiation limited to patients with four or more positive nodes, as recommended in international consensus reports? A subgroup analysis of the DBCG 82 b\&c randomized trials. Radiother Oncol. 2007;82(3):247-53.

3. McGale P, Taylor C, Correa C, et al. Effect of radiotherapy after mastectomy and axillary surgery on 10-year recurrence and 20-year breast cancer mortality: meta-analysis of individual patient data for 8135 women in 22 randomised trials. Lancet. 2014;383(9935):2127-35.

4. Recht A, Comen EA, Fine RE, et al. Postmastectomy radiotherapy: An American Society of Clinical Oncology, American Society for Radiation Oncology, and Society of Surgical Oncology Focused Guideline Update. J Clin Oncol. 2016;34(36):4431-42.

5. Chagpar AB. Debate: Postmastectomy Radiation Therapy in T1/ 2N1 Disease. Ann Surg Oncol. 2021. https://doi.org/10.1245/s104 34-021-10500-5.

Publisher's Note Springer Nature remains neutral with regard to jurisdictional claims in published maps and institutional affiliations. 